\title{
Evaluation of colour change and surface roughness of two resin-based composites when exposed to beverages commonly used by children: an in-vitro study
}

\author{
G. Elwardani ${ }^{1} \cdot$ A. A. Sharaf ${ }^{1} \cdot$ A. Mahmoud ${ }^{1}$
}

Received: 5 April 2017 / Accepted: 29 November 2018 / Published online: 26 December 2018

(c) The Author(s) 2018

\begin{abstract}
Aim To evaluate and compare the surface roughness and colour change of microhybrid and nanocomposite after exposure to beverages commonly used by children.

Methods Thirty discs were prepared using Filtek Z250 and Filtek Supreme composites. Discs were immersed in distilled water, orange juice and Coca-Cola. Colour and surface roughness were measured at baseline and on days 15 and 30.

Results Filtek Z250 and Filtek Supreme showed no significant difference in surface roughness and colour change at all measurement times. Immersion solution had a significant effect on surface roughness as well as colour change. Specimens immersed in Coca-Cola demonstrated the highest effect, followed by those immersed in orange juice, while specimens immersed in distilled water had the least effect. There was a significant increase in surface roughness and colour change over time. The change in colour was more evident in the period between baseline measurements and after 15 days. There was a significant interaction between media type and time.

Conclusions Both composites showed no significant difference in roughness and colour change at all measurement times. There was a significant increase in surface roughness and colour change in all immersion solutions tested over time. CocaCola caused unacceptable colour change.
\end{abstract}

Keywords Colour change $\cdot$ Surface roughness $\cdot$ Filtek Z250 $\cdot$ Filtek supreme $\cdot$ Beverages $\cdot$ Children

\section{Introduction}

A common problem confronting paediatric dentists is the management of carious/fractured anterior teeth. The clinician must consider the longevity of the anterior restoration, as well as, its post-operative aesthetic appearance (Donly and Dean 2016). A well recognised factor for satisfactory anterior restorations is the correct colour match. Hence; the restorative material must not only provide an initial shade

G. Elwardani

gwardany@hotmail.com

A. A. Sharaf

dr.alysharaf@hotmail.com

A. Mahmoud

d.amelmahmoud@hotmail.com

1 Pediatric Dentistry and Dental Public Health Department, Faculty of Dentistry, Alexandria University, 18 El Salam st, Sidigaber 2nd Floor, Apt 8, P.O. Box: 21523, Alexandria, Egypt match, but must also maintain an acceptable appearance (Mohan et al. 2008).

Resin-based composites are now used principally for direct aesthetic restorations with great success and high patient acceptance. Enhanced technology has led to the development of microhybrid composites (particle size $<1 \mu \mathrm{m}$ ) with good mechanical, physical and aesthetic properties (Powers et al. 2017). Recently, the development of nanotechnology allowed for the production of particle sizes of $0.1-100$ nanometer $(1 \mathrm{~nm}=1 / 1000 \mu \mathrm{m})$. The introduction of nano-sized filler particles resulted in slight enhancement of the mechanical and physical properties, while the most notable improvement was in the optical properties. This can be explained by the small diameter of nanofillers which is a fraction of the wavelength of visible light (400-750 nm), thus the human eye cannot detect these particles. Consequently, manufacturers now recommend the use of microhybrid and nanocomposite. They are now well accepted as universal composites as they can be used for both anterior and posterior teeth (Mitra et al. 2003). 
The physical properties of composites are influenced by the oral environment, which is exposed to a variety of media that may stain or alter the restoration surface. Discolouration is a frequent reason for replacement of dental restorations. Samorodnitzky-Naveh et al. (2007) observed that tooth colour was the primary reason for dental dissatisfaction in $89 \%$ of patients. Composite discolouration is multifactorial, it can be intrinsic and/or extrinsic. Intrinsic factors involve the discolouration of the resin material itself, such as the alteration of the resin matrix and/or the resin-filler interface. Humidity, thermal changes and exposure to ultraviolet radiation contribute to the intrinsic colour change (Oysaed and Ruyter 1986). Where as, extrinsic factors for discolouration is caused by adsorption of colourants from exogenous sources. The size and shape of filler particles have a direct impact on the surface roughness and susceptibility to extrinsic staining (Curtin et al. 2008). Rough composite causes plaque accumulation, which may lead to gingival and periodontal inflammation. Moreover, it increases the scattering of light striking on the restorative material which impairs its aesthetic appearance (Mohan et al. 2008).

Various in vitro studies have investigated the discolouration of dental restorations as a result of prolonged exposure to daily beverages, mouthwash, ultraviolet radiation (Sarafianou et al. 2007; Celik et al. 2008; Curtin et al. 2008). Topcu et al. (2009) evaluated the discolouration of Filtek Z250 and Filtek Supreme of shade A2 after 1 day of immersion in different solutions (artificial saliva, carrot juice, lemon juice, cherry juice, Coca-Cola, coffee and red wine). The least colour change was observed with Filtek Supreme and artificial saliva; whereas the most colour change was observed with Filtek Z250 and red wine. Al-Shalan (2009) compared the colour change of Filtek Z250 and Filtek Supreme exposed to Coca-Cola at baseline, after $48 \mathrm{~h}$, and after 2 weeks. Filtek Z250 showed the least colour change and remained clinically acceptable at all times. After 2 weeks, values reported for Filtek Supreme were clinically unacceptable. The results of these studies are controversial.

While many studies existed on the staining effects of beverages on composite restorations, the most tested beverages were tea, coffee and wine, which are normally associated with adult tooth stains. Only a few studies have evaluated the effect of common beverages ingested by children (Mohan et al. 2008). Galal (2002) described the food patterns in Egypt through analysis of the national food consumption surveys in both urban and rural areas. A $24 \mathrm{~h}$ recall of frequency and quantitative food intake for one child, age ranged between 2 and 18 years, were collected. Results showed that tea and carbonated soft drinks were the most commonly consumed beverages. Frary et al. (2004) studied the dietary pattern among children aged 6-17 years in the United States of America who participated in the national food survey. The study sample was subgrouped into children aged 6-11 years and adolescents aged 12-17 years. As children progressed from childhood to adolescence, the prevalence and frequency of milk and fruit juice intake decreased, whereas soft drink intake was tripled.

It is, therefore, important to study the effect of prolonged exposure of beverages commonly consumed by children, such as fruit juices and soft drinks, on the colour change and surface roughness of composite restorative materials.

\section{Materials and methods}

Thirty composite discs $(1 \mathrm{~cm}$ diameter $\times 2 \mathrm{~mm}$ thick $)$ were prepared from each composite resin material; Filtek Z250 A1 and Filtek Supreme XT A1B (3M ESPE, USA) using a specially designed metal mould. It consisted of a plastic base, two split metal plates and a plastic cover. A transparent mylar strip (Kerr, USA) was placed between the plastic base and the metal plates; below the mould. According to the manufacturer, the transparent strip is $10 \mathrm{~cm}$ in long, $1 \mathrm{~cm}$ wide and $0.05 \mathrm{~mm}$ thick. After the resin-based composite material was packed and overfilled into the mould, another mylar strip was placed above the metal plate. The plastic cover was placed above the metal plate with a weight of $1.5 \mathrm{~g}$ placed over it for $30 \mathrm{~s}$ to allow for compact voidless specimens and to extrude excess material (Badra et al. 2005). After the excess composite material was removed, specimens were polymerised using a LED light curing unit (BluePhase, Ivoclar Vivadent Inc., USA; light intensity of $1200 \mathrm{~mW} / \mathrm{cm}^{2}$ ) for $20 \mathrm{~s}$ from the top and bottom surfaces. The tip of the light source was placed in contact with the plastic base and cover, which had the same thickness. Hence, the distance between the light source and the specimen was standardised. All composite discs were visually inspected for surface defects and those with visible flaws were discarded. These defects resulted from inadequate material or inadequate packing force. The prepared samples represented the two main groups $(n=30)$, classified according to the type of resin used; Group I: assigned for Filtek Z250 (microhybrid composite) and Group II: assigned for Filtek Supreme (nanocomposite).

Each main group was further subdivided into 3 subgroups $(n=10)$, classified according to the type of immersion liquid used; Subgroup A (control): assigned for discs immersed in distilled water (Runyes water distiller, China), Subgroup B: assigned for discs immersed in orange juice, (Juhayna, Egypt) and Subgroup C: assigned for discs immersed in Coca-Cola (Coca-Cola, Egypt). The containers - where samples were kept - were ticket coded as follows: Latin numbers (I and II): representing the two main groups, Letters (A, B, and C): representing the subgroups and Numbers $(1,2, \ldots 10)$ : representing the number of specimen in each 
subgroup. The coding system was developed for double blinding during laboratory measurements and statistical evaluation.

After the composite discs were removed from the mould, round bur size 1 (Dentsply, USA) was used to mark an indent along the thickness of the specimens. Half the tip of the bur ( $0.8 \mathrm{~mm}$ diameter) was drilled at the six opposite points along the diameter of the specimen. Points $\mathrm{A}$ and $\mathrm{B}$ were marked where the two split metal plates meet. The circumference of the specimen was divided into four equal quarters and an indent was marked arbitrarily along the thickness of the specimen in the middle of each quarter. These four indents were named $\mathrm{C}, \mathrm{D}, \mathrm{E}$ and $\mathrm{F}$ respectively in a clockwise direction. Hence, three lines along the diameter of each specimen namely $\mathrm{AB}, \mathrm{CE}$ and $\mathrm{DF}$ represented standardised reference lines for measuring the surface roughness.

Composite discs were distributed into coded petri dishes. Firstly, the composite discs were stored in $20 \mathrm{ml}$ of saliva natura (Medac, UK) at $37{ }^{\circ} \mathrm{C}$ for $24 \mathrm{~h}$ in an incubator to ensure stabilisation of monomer conversion and to mimic oral conditions (Oysaed and Ruyter 1986). Secondly, the petri were removed from the incubator, the existing liquid was drained and the composite discs were washed thoroughly. Washing was done by rinsing the composite discs in distilled water for $10 \mathrm{~s}$. Thirdly, the composite discs were reimmersed in $20 \mathrm{ml}$ of the immersion liquids and placed back in the incubator at $37{ }^{\circ} \mathrm{C}$ for 30 days. The immersion liquids represent the three subgroups i.e. distilled water, orange juice and Coca-Cola. The same rinsing process was repeated every day during the incubation period and the three immersion liquids were refreshed to prevent microbial growth. A digital pH meter (Hanna Instruments, Model 211, USA) was used to measure the $\mathrm{pH}$ of the fresh immersion liquids daily and the average $\mathrm{pH}$ was calculated after 30 days. All specimens were carefully handled from the sides to prevent any scratches to the surface (Nagem Filho et al. 2003).

\section{Colour evaluation}

The colour of each specimen was measured using a Spectrophotometer (Shimadzu 3101 PC, Japan) before exposure (at baseline), on day 15 and on day 30 of exposure. The colour measurements were performed according to the International Commission on Illumination (usually abbreviated as CIE for its French name, Commission internationale de l'éclairage). Colour was expressed by the CIE-Lab system. The symbol $\mathrm{L}^{*}$ refers to the measurement along the white-black axis, $\mathrm{a}^{*}$ refers to the measurement along the red-green axis and $b^{*}$ refers to the measurement in the yellow-blue axis. The total colour change $(\Delta \mathrm{E})$ was calculated using the equation: $\Delta \mathrm{E}$
$=\left[(\Delta \mathrm{L})^{2}+(\Delta \mathrm{a})^{2}+(\Delta \mathrm{b})^{2}\right]^{1 / 2}$, in which $\Delta \mathrm{L}, \Delta \mathrm{a}$ and $\Delta \mathrm{b}$ are the differences in colour values before and after immersion in the tested liquids (CIE 1971). Between measurements and during transportation samples were kept in dry and dark conditions to prevent ageing of composite (Sarafianou et al. 2007). Three successive readings were taken on each surface for every specimen, and the average value was recorded.

\section{Surface roughness evaluation}

Each specimen was measured following the same time sequence (at baseline, day 15 and day 30) with Surtronic 2 (Taylor and Hobsons, UK). Surface roughness (Ra) is defined as the average vertical deviation from mean line along the surface of the specimen; it is measured in micrometer $(\mu \mathrm{m})$. A diamond stylus was moved perpendicular to the surface along the diameter of the composite discs. The vertical movement of the stylus, as it ascended or descended over the irregularities of the specimen, was converted into digital readings. Three successive measurements were taken on each surface and the average value was recorded (Cazzaniga et al. 2015).

\section{Null hypothesis}

This study was performed to investigate and compare the colour change and surface roughness of 2 composite resins (Filtek Z250 and Filtek supreme) in different immersion solutions (distilled water, orange juice and Coca-Cola) over time (at baseline, after 15 and 30 days). The null hypothesis is there will be no change in colour and surface roughness for Filtek Z250 and Filtek supreme at baseline and after immersion in the tested beverages for 15 and 30 days.

\section{Statistical analysis}

Data was analysed using SPSS (Statistical Package for Social Science) program version 21 (IBM Corp. 2012). Descriptive statistics were calculated as minimum-maximum, median, mean and standard deviation. Independent sample $t$-test was used to compare between the two main groups at different times of measurement. Independent one-way Analysis of Variance (ANOVA) was carried out between the subgroups at each measurement time. Twoways repeated measures ANOVA was carried out for time related variables. Pair-wise comparison after ANOVA was carried out using the Games-Howell method (Field 2013). Partial Eta squared was considered according to Cohen's guidelines (Pierce et al. 2004). In the present study, an alpha level was set to 5\% with a significant level of $95 \%$ 
and a beta error accepted up to $20 \%$ with a power of study of $80 \%$. Graphically, the data is presented in a box-andwhiskers plot. The box represents the inter-quartile range (from 25th to 75 th percentiles). The thick line in the middle of the box represents the median. The whiskers represent the minimum and maximum values after excluding outliers and extremes which are represented as the blackfilled circles and asterisks, respectively.

\section{Results}

\section{Colour evaluation}

The mean values and standard deviations of the colour change $(\Delta \mathrm{E})$ are presented in Table 1 and graphically represented by Figs. 1, 2 and 3 respectively. Independent sample t-test showed no significant difference between Filtek Z250 and Filtek Supreme at all times of measurements (baseline: $\mathrm{p}=0.53$-after 15 days: $\mathrm{p}=0.56$-after 30 days: $\mathrm{p}=0.39)$. Thus, there is significant evidence to accept the null hypothesis. In both groups, ANOVA revealed a significant difference between the different immersion solutions at all times of measurements. Filtek Z250 specimens had $p=0.00$ at all times. Filtek Supreme group showed $p=0.00$ at baseline and after 30 days along with $p=0.01$ after 15 days. Specimens immersed in Coca-Cola had the most change in colour, followed by those immersed in orange juice, while specimens immersed in distilled water had the least change. Therefore, the null hypothesis was

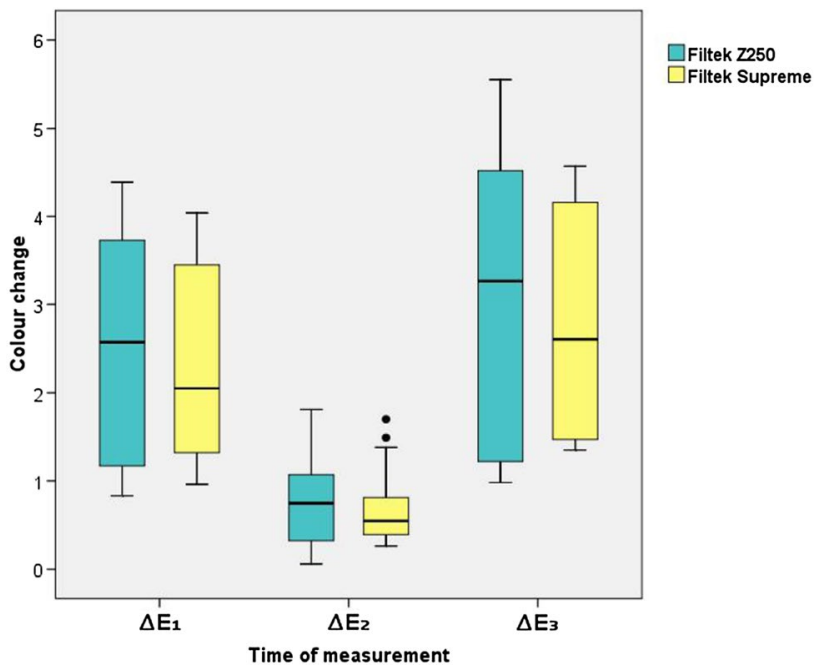

$\Delta \mathrm{E}_{1}$ : Colour change between measuremets at baseline and after 15 days $\Delta \mathrm{E}_{2}$ : Colour change between measurements at 15 days and 30 days $\Delta \mathrm{E}_{3}$ : Colour change measurments between baseline and after 30 days

Fig. 1 Box and whisker plot of the colour change for two composite materials with regards to time

rejected. Repeated measures ANOVA revealed that colour change increased significantly over time. There was a significant time effect as regards the composite material investigated, Pillai's Trace $=0.80, \mathrm{~F}_{(2,57)}=115.27$, $\mathrm{p}=0.00$, partial Eta squared $\left(\eta^{2}\right)=0.80$, power $=1.00$. Moreover, there was a significant time effect as regards the immersion solutions tested, Pillai's Trace $=0.98$,
Table 1 Colour change values for the two composite materials with regards to immersion liquids and time

\begin{tabular}{llll}
\hline & $\Delta \mathrm{E}_{1}$ & $\Delta \mathrm{E}_{2}$ & $\Delta \mathrm{E}_{3}$ \\
\hline Filtek Z250 $(\mathrm{n}=30)$ & & & \\
$\quad$ Distilled water & $1.05 \pm 0.15^{\mathrm{a}}$ & $0.23 \pm 0.14^{\mathrm{a}}$ & $1.13 \pm 0.11^{\mathrm{a}}$ \\
Orange juice & $2.56 \pm 0.21^{\mathrm{b}}$ & $0.74 \pm 0.21^{\mathrm{b}}$ & $3.26 \pm 0.10^{\mathrm{b}}$ \\
Coca-Cola & $3.87 \pm 0.27^{\mathrm{c}}$ & $1.25 \pm 0.40^{\mathrm{c}}$ & $4.98 \pm 4.39^{\mathrm{c}}$ \\
Total & $2.49 \pm 1.19$ & $0.74 \pm 0.50$ & $3.12 \pm 1.63$ \\
Comparison among the three media sub- & $\mathrm{F}_{(2,27)}=426.53$ & $\mathrm{~F}_{(2,27)}=35.14$ & $\mathrm{~F}_{(2,27)}=523.93$ \\
groups within Filtek Z250 group & $\mathrm{p}=0.00^{*}$ & $\mathrm{p}=0.00^{*}$ & $\mathrm{p}=0.00^{*}$ \\
Filtek Supreme (n=30) & & & \\
Distilled water & $1.27 \pm 0.18^{\mathrm{a}}$ & $0.40 \pm 0.08^{\mathrm{a}}$ & $1.43 \pm 0.07^{\mathrm{a}}$ \\
Orange Juice & $2.12 \pm 0.68^{\mathrm{b}}$ & $0.80 \pm 0.43^{\mathrm{b}}$ & $2.63 \pm 0.12^{\mathrm{b}}$ \\
Coca-Cola & $3.54 \pm 0.34^{\mathrm{c}}$ & $0.82 \pm 0.38^{\mathrm{b}}$ & $4.33 \pm 0.16^{\mathrm{c}}$ \\
Total & $2.31 \pm 1.05$ & $0.67 \pm 0.37$ & $2.80 \pm 1.21$ \\
Comparison among the three media sub- & $\mathrm{F}_{(2,27)}=65.04$ & $\mathrm{~F}_{(2,27)}=5.03$ & $\mathrm{~F}_{(2,27)}=1377.81$ \\
groups within Filtek Supreme group & $\mathrm{p}=0.00^{*}$ & $\mathrm{p}=0.01^{*}$ & $\mathrm{p}=0.00^{*}$ \\
Comparison between the two groups & $\mathrm{t}=0.63$ & $\mathrm{t}=0.59$ & $\mathrm{t}=0.87$ \\
Independent sample t-test & $\mathrm{p}=0.53 \mathrm{NS}$ & $\mathrm{P}=0.56 \mathrm{NS}$ & $\mathrm{p}=0.39 \mathrm{NS}^{\circ}$ \\
\hline
\end{tabular}

$\Delta \mathrm{E} 1$ : colour change between measurements at baseline and after 15 days

$\triangle \mathrm{E} 2$ : colour change between measurements at 15 days and 30 days

$\Delta \mathrm{E} 3$ : colour change measurements between baseline and after 30 days

Different superscripts indicate significant difference $(p<0.05)$ 


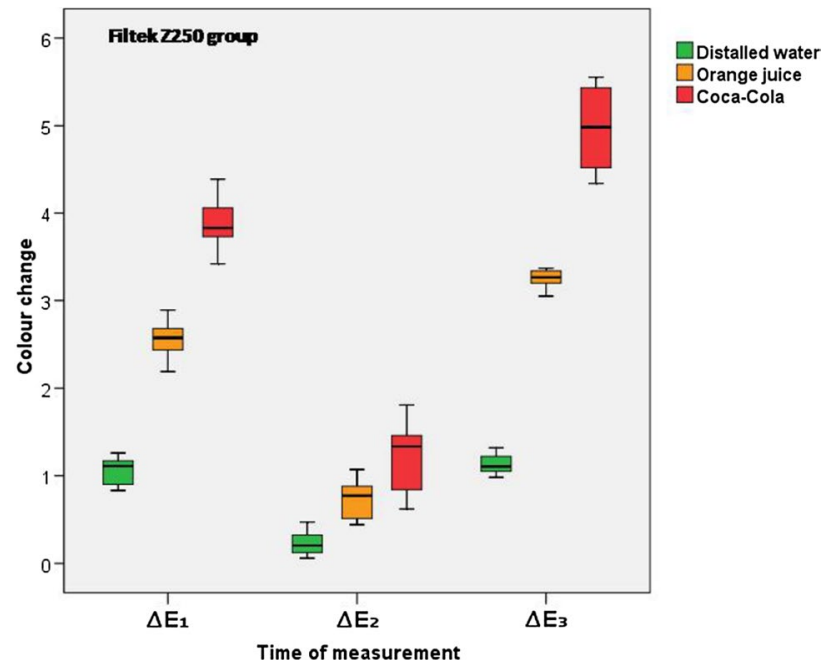

$\Delta \mathrm{E}_{1}$ : Colour change between measuremets at baseline and after 15 days $\Delta \mathrm{E}_{2}$ : Colour change betweemeasurements at 15 days and 30 days $\Delta \mathrm{E}_{3}$ : Colour change measurements between baseline and after 30 days

Fig. 2 Box and whisker plots of the colour change for Filtek Z250 group with regards to immersion liquids and time

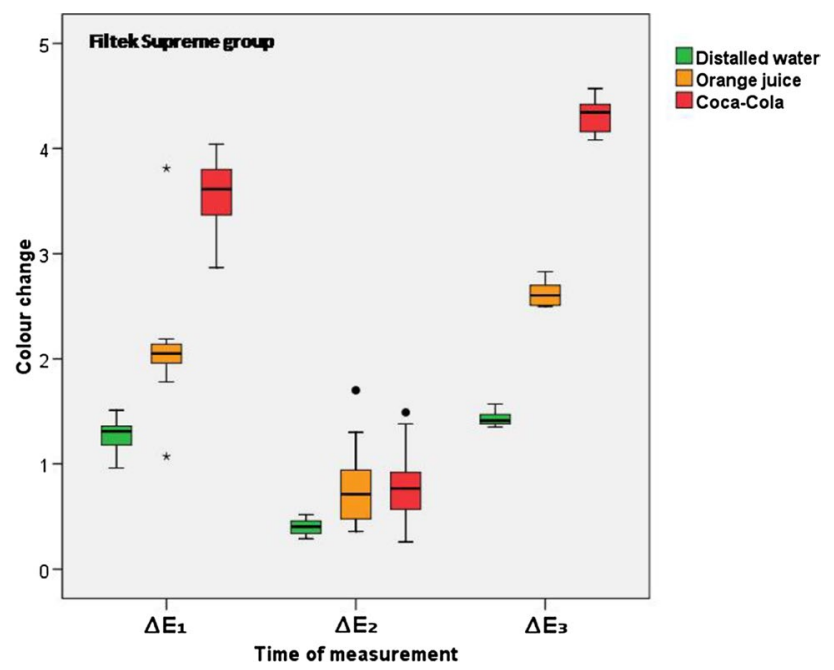

$\Delta \mathrm{E}_{1}$ : Colour change between masurements at baseline and after 15 days $\Delta \mathrm{E}_{2}$ : Colour change between measurements at 15 days and 30 days $\Delta \mathrm{E}_{3}$ : Colour change measurements between baselinend after 30 days

Fig. 3 Box and whisker graph of the colour change for Filtek Supreme group with regards to immersion liquids and time

$\mathrm{F}_{(2.56)}=1483.20, \mathrm{p}=0.00$, partial Eta squared $\left(\eta^{2}\right)=0.98$, power $=1.00$. Follow-up (post-hoc) comparisons indicated that each pair-wise difference was significant $p=0.00$ regarding the composite material investigated and the immersion solution tested. The change in colour was more evident in the period between baseline measurements and after 15 days $\left(\Delta \mathrm{E}_{1}>\Delta \mathrm{E}_{2}\right)$. Thus, there is significant evidence to reject the null hypothesis.

\section{Surface roughness evaluation}

The mean values and standard deviations of the surface roughness measured in micrometers are presented in Table 2 and graphically represented by Figs. 4, 5 and 6 respectively. Independent sample t-test showed no significant difference between Filtek Z250 and Filtek Supreme at all time measurements (baseline: $p=0.59$-after 15 days: $p=0.39$-after 30 days: $p=0.14$ ). Thus, there is significant evidence to accept the null hypothesis. In both groups, ANOVA revealed a significant difference between the different immersion solutions at all times of measurement. At baseline, Filtek Z250 specimens showed $p=0.01$ along with $p=0.03$ in Filtek Supreme group. Both groups showed $p=0.00$ after 15 and 30 days. Specimens immersed in Coca-Cola had the roughest surface, followed by those immersed in orange juice, while specimens immersed in distilled water had the least surface roughness. Therefore, the null hypothesis was rejected. Repeated measures ANOVA revealed that there was a significant increase in roughness over time. There was a significant time effect as regards the composite material investigated, Pillai's Trace $=0.87, \mathrm{~F}_{(2,57)}=193.50, \mathrm{p}=0.00$, partial Eta squared $\left(\eta^{2}\right)=0.87$, power $=1.00$. Moreover, there was a significant time effect as regards the immersion solutions tested, Pillai's Trace $=0.99, \mathrm{~F}_{(2,56)}=3591.46, \mathrm{p}=0.00$, partial Eta squared $\left(\eta^{2}\right)=0.99$, power $=1.00$. Follow-up (posthoc) comparisons indicated that each pair-wise difference was significant $p=0.00$ regarding the composite material investigated and the immersion solution tested. Thus, there is significant evidence to reject the null hypothesis.

In general, there was a significant interaction between media type and time, Pillai's Trace $=0.94, \mathrm{~F}_{(4,114)}=25.26$, $\mathrm{p}=0.00$, partial Eta squared $\left(\eta^{2}\right)=0.47$, power $=1.00$.

\section{Discussion}

In the present study, composite shade A1 was investigated because it is the most commonly used shade in paediatric dentistry. This is in accordance with Kim et al. (2007) who evaluated the colour frequency of primary teeth using Vita intra-oral spectrophotometer. It was concluded that shade A1 was the best match (46\%), followed by A2 (25\%) and B2 (11\%).

According to the manufacturer, the thickness of composite discs should be limited to $2 \mathrm{~mm}$ as it is the accepted thickness for the incremental technique of composite application. A matrix strip was used in this study to mimic the clinical procedures for restoring anterior primary teeth with strip crowns and because it provides the smoothest surface 
Table 2 Surface roughness values $(\mu \mathrm{m})$ for the two composite materials with regards to different immersion liquids and time

\begin{tabular}{llll}
\hline & Baseline & 15 days & 30 days \\
\hline Filtek Z250 $(\mathrm{n}=30)$ & & & \\
Distilled water & $0.17 \pm 0.01^{\mathrm{a}}$ & $0.51 \pm 0.05^{\mathrm{a}}$ & $0.90 \pm 0.04^{\mathrm{a}}$ \\
Orange juice & $0.14 \pm 0.03^{\mathrm{b}}$ & $0.81 \pm 0.05^{\mathrm{b}}$ & $1.00 \pm 0.05^{\mathrm{b}}$ \\
Coca-Cola & $0.15 \pm 0.02^{\mathrm{b}}$ & $1.10 \pm 0.03^{\mathrm{c}}$ & $1.98 \pm 0.04^{\mathrm{c}}$ \\
Total & $0.15 \pm 0.02$ & $0.81 \pm 2.50$ & $1.29 \pm 0.50$ \\
Comparison among the three media sub- & $\mathrm{F}_{(2,27)}=5.56$ & $\mathrm{~F}_{(2,27)}=437.57$ & $\mathrm{~F}_{(2,27)}=1955.34$ \\
groups within Filtek Z250 group & $\mathrm{p}=0.01^{*}$ & $\mathrm{p}=0.00^{*}$ & $\mathrm{p}=0.00^{*}$ \\
Filtek Z250 (n=30) & & & \\
Distilled water & $0.14 \pm 0.02$ & $0.46 \pm 0.04^{\mathrm{a}}$ & $0.86 \pm 0.04^{\mathrm{a}}$ \\
$\quad$ Orange juice & $0.16 \pm 0.01$ & $0.76 \pm 0.08^{\mathrm{b}}$ & $0.96 \pm 0.05^{\mathrm{b}}$ \\
Coca-Cola & $0.13 \pm 0.03$ & $1.04 \pm 0.06^{\mathrm{c}}$ & $1.58 \pm 0.08^{\mathrm{c}}$ \\
Total & $0.15 \pm 0.02$ & $0.75 \pm 0.25$ & $1.13 \pm 0.33$ \\
Comparison among the three media sub- & $\mathrm{F}_{(2,27)}=3.83$ & $\mathrm{~F}_{(2,27)}=216.44$ & $\mathrm{~F}_{(2,27)}=403.67$ \\
$\quad$ groups within Filtek Supreme group & $\mathrm{p}=0.03^{*}$ & $\mathrm{p}=0.00^{*}$ & $\mathrm{p}=0.00^{*}$ \\
Comparison between the two groups & $\mathrm{t}=0.55$ & $\mathrm{t}=0.87$ & $\mathrm{t}=1.49$ \\
Independent-Sample t-test & $\mathrm{p}=0.59 \mathrm{NS}$ & $\mathrm{p}=0.39 \mathrm{NS}$ & $\mathrm{p}=0.14 \mathrm{NS}^{\mathrm{n}}$ \\
\hline
\end{tabular}

Different superscripts indicate significant difference $(p<0.05)$

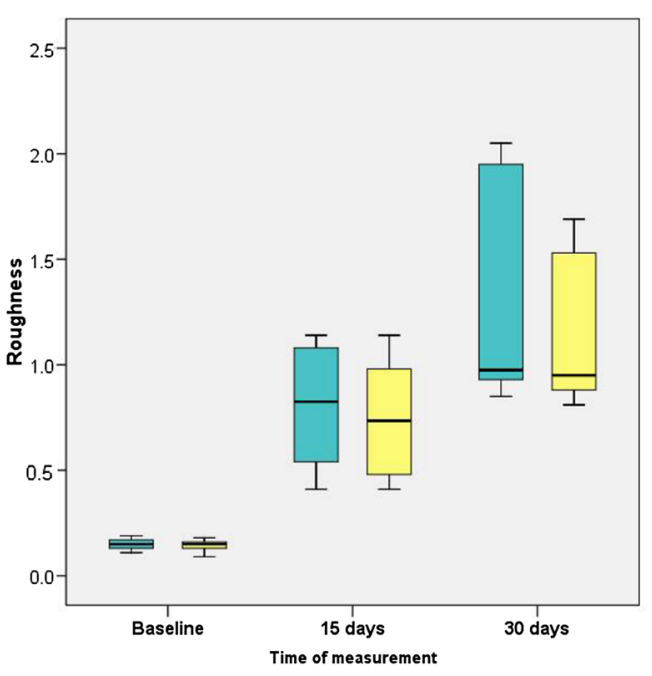

Fig. 4 Box and whisker plot of the surface roughness $(\mu \mathrm{m})$ for two composite materials with regards to time

when compared to other finishing and polishing procedures. Moreover, finishing and polishing procedures cannot be standardised for all composite discs regarding the direction, force and time per stroke (Sarac et al. 2006).

Coding was done to the container, where samples were kept, rather than the samples itself to avoid any interference with the surface roughness and colour measurements.

Similar to Erdemir et al. (2013), distilled water was utilised in the present study as the control group instead of artificial saliva. The authors justified such choice as artificial saliva is not considered to be a better clinical alternative to distilled water. Also, Turssi et al. (2002) concluded that there was no statistically significant difference in surface

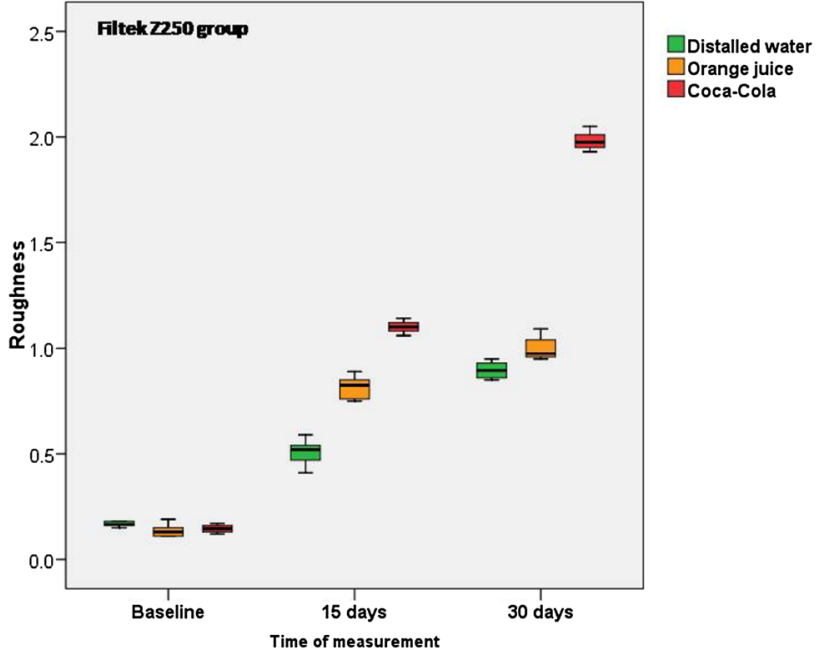

Fig. 5 Box and whisker plot of the surface roughness $(\mu \mathrm{m})$ for Filtek $\mathrm{Z} 250$ group with regards to immersion liquids and time

roughness of four resin-based restorative materials after storage in distilled water and artificial saliva. Moreover, immersion in saliva natura may cause biased colour results as it contains mucin, which is yellowish in colour (Omata et al. 2006). Additionally, immersion in other artificial saliva preparations may affect the surface roughness due to its potential remineralisation properties (Ionta et al. 2014).

The immersion duration of the present study was determined according to the literature review by Ferracane (2006), who deduced that the sufficient immersion time for most composites to reach saturation was in the range between 7 and 60 days. 


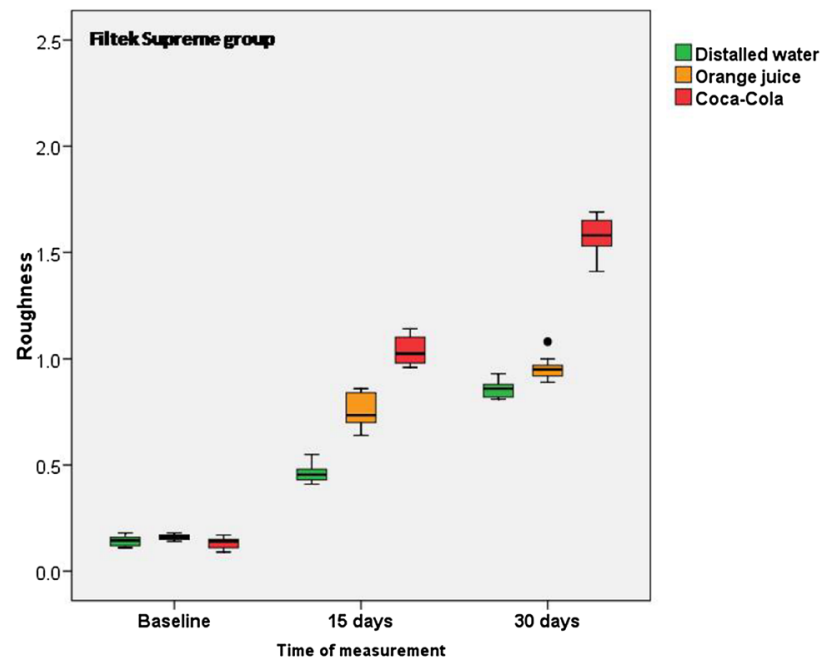

Fig. 6 Box and whisker plot of the surface roughness $(\mu \mathrm{m})$ for Filtek Supreme group with regards to immersion liquids and time

Besides visual assessment, colour determination in dentistry can be performed instrumentally using a spectrophotometer which compares specimens against a standard illuminant. It eliminates subjective errors and more importantly it is more precise than the naked eye in measuring slight differences (Joiner 2004). The CIE strongly recommended using the equation $\Delta \mathrm{E}=\left[(\Delta \mathrm{L})^{2}+(\Delta \mathrm{a})^{2}+(\Delta\right.$ b) $\left.{ }^{2}\right]^{1 / 2}$ rather than comparing the 3 colour vectors ( $\mathrm{L}^{*}, \mathrm{a}^{*}$ and $\mathrm{b}^{*}$ ) to evaluate colour change. $\Delta \mathrm{E}$ is more informative as it summarises the difference in the 3 colour vectors and represents their combined effect on colour change (CIE 1971).

Various methods can be used to evaluate surface roughness, which is an important factor influencing colour stability and biofilm formation. Qualitative evaluation includes atomic force microscopy (AFM) and scanning electron microscopy (SEM), whereas, quantitative evaluation includes contact diamond and non-contact laser profile analysis. The most widely used and accepted method is probably contact stylus profilometry (Cazzaniga et al. 2015).

The results of the present study showed that colour change increased significantly for all immersion liquids over time. There was more colour change between the baseline measurements and after 15 days than that between 15 and 30 days for both composite materials used. This agrees with Oysaed and Ruyter (1986), who stated that most water sorption was observed during the first week. It is reported that stain sorption is closely related to water sorption, which is caused by a high resin content and lack of coupling agent (Dietschi et al. 1994).

According to the manufacturer, the organic matrix in Filtek Z250 is composed of Bis-GMA, UDMA and Bis-EMA with inorganic filler loading of $60 \%$ by volume $(82 \%$ by weight) in the form of zirconia/silica of particle size range of $0.01-3.5 \mu \mathrm{m}$ and an average particle size of $0.6 \mu \mathrm{m}$ without silane treatment. While the organic matrix in Filtek Supreme is composed of Bis-GMA, Bis-EMA, UDMA and TEGDMA and with inorganic filler loading of $59.5 \%$ by volume $(78.5 \%$ by weight) in the form of zirconia/silica with primary particle size of 5-20 nm aggregated into secondary nanoclusters of particle size range $0.6-1.4 \mu \mathrm{m}$.

The results of the present study showed no significant difference in colour change between Filtek Z250 and Filtek Supreme at all time measurements. Such results disagree with the findings of Topcu et al. (2009), who reported that Filtek Z250 had a significantly greater colour change than Filtek Supreme. This finding was explained by the nano filler size in Filtek Supreme demonstrating less surface roughness and consequently less adsorption of colour. However, this explanation is not in agreement with Gönülol and Yilmaz (2012) who concluded that composites with smaller filler size did not necessarily show lower discolouration.

The colour results of the present study also oppose the findings of Ertaş et al. (2006) and Al-Shalan (2009). Both authors concluded that Filtek supreme had a significantly greater colour change than Filtek Z250. It was explained by the presence of the hydrophilic TEGDMA in Filtek supreme which permitted more water/stain sorption and thus more discolouration. According to the manufacturer, this justification does not comply with the advantages of adding TEGDMA to the resin matrix. It is mentioned that adding TEGDMA causes higher conversion of double bonds during polymerisation. Sideridou et al. (2002) compared that the degree of conversion of different composite resins and arranged them in the following order: TEGDMA $>$ UDMA > Bis-EMA > Bis-GMA. The high degree of crosslinking creates a harder resin matrix which is more resistant to absorption of colourants. Also, according to the manufacturer, the absence of silane treatment for Filtek Z250 is likely to cause more water/stain sorption.

Such inconsistent colour results can be justified by the small number of specimens tested, the short duration of immersion and the variation in finishing/polishing techniques used.

The ageing of composite resins is an outcome of combined factors in the oral cavity such as $\mathrm{pH}$ and temperature variations, exposure to mechanical forces as well prolonged storage in water/ethanol (Çelik et al. 2011). Considering that immersion of composite resin in different solutions is part of the ageing process (de Moraes et al. 2008); hence, the colour results of the present study agree with Gerhardt et al. (2013). The author concluded that there was no significant difference in colour change between Filtek Z250 and Filtek Supreme after accelerated aging.

In the present study, specimens immersed in distilled water had the least change, followed by those immersed in 
orange juice, while specimens immersed in Coca-Cola had the most change in colour. The CIE correlated $\Delta \mathrm{E}$ with limits of human detection. It concluded that value of $\Delta \mathrm{E}<1$ is regarded as not perceptible by the human eye. Values $1<$ $\Delta \mathrm{E}<3.3$ is considered perceptible by skilled operators, but still clinically acceptable. Value of $\Delta \mathrm{E}>3.3$ is considered perceptible by non-skilled personnel, hence, clinically not acceptable.

In the present study, the colour change of composite discs immersed in distilled water (average $\Delta \mathrm{E}_{3}=1.28$ ) and orange juice (average $\Delta \mathrm{E}_{3}=2.95$ ) after 30 days showed clinically acceptable colour change, while those immersed in CocaCola (average $\Delta \mathrm{E}_{3}=4.66$ ) were clinically unacceptable.

Previous studies demonstrated statistically significant colour change after immersion of composite resin in artificial saliva and distilled water (Çelik et al. 2011; Uchimura et al. 2014). Although distilled water has no colourants, the colour change is a result of water sorption into the resin matrix (Venz and Dickens 1991). Sideridou et al. (2002) reported that the hydrophilicity of resin is in the following order: TEGDMA $>$ Bis-GMA $>$ UDMA and Bis-EMA.

Mainly, water sorption occurs by direct absorption into the resin matrix and diffuses into the microvoids within the polymer. Secondly, water adsorb on the filler surface - as the inorganic particles are incapable of absorbing water molecule-accumulating at the filler-matrix interface (Curtis et al. 2008). Water sorption hydrolyses the chemical bond between the resin matrix and filler particle causing filler dislodgment and microcracks formation (Söderholm et al. 1984). The loss of fillers leads to a rough surface that is easily eroded. The microcracks allow for further water diffusion, which acts as a vehicle for stain penetration; hence causing discolouration (Ferracane 2006).

The colour findings of the present study can be explained by the differences in acidity among the tested immersion liquids, in which the measured $\mathrm{pH}$ for distilled water was 6.9, where as orange juice possessed a $\mathrm{pH}$ of 3.5 and Coca-Cola is presented by a pH of 2.6 .

Coca-Cola ( $\mathrm{pH}$ 2.6) is strongly implicated to colour change of composite due to the presence of brown pigments, phosphoric acid and carbon dioxide, which is transformed into carbonic acid. Such strong acidity degrades/softens the composite material and erodes the surface, hence, increasing its roughness. This leads to penetration of colourants into the organic phase as well as adsorption of pigments on the composite surface (Ghiorghe et al. 2013). Accordingly, orange juice ( $\mathrm{pH} 3.5$ ) causes less discolouration as it contains yellow pigments and the relatively weaker citric acid (Hotwani et al. 2014).

In conclusion, the results of this study agree with Poggio et al. (2016) in that the long term exposure to some oral pigments can significantly affect the colour stability of modern aesthetic restorative materials regardless of materials' different composition.

According to the results of this present study, there was a significant increase in surface roughness and colour change over time. This was in agreement with Bansal et al. (2012) and Lepri and Palma-Dibb (2012) in which, results showed that immersion time was directly proportional with colour change and surface roughness.

The results of the present study showed no significant difference in surface roughness between Filtek Z250 and Filtek Supreme at all measurement times. This finding is supported by Korkmaz et al. (2008) and Penteado et al. (2010). The former authors concluded that there was no significant difference in surface roughness between Filtek Z250 and Filtek Supreme using matrix strips and after applying different polishing systems. While the latter authors showed that there was no difference in surface roughness between Filtek Z250 and Filtek Supreme at baseline, after $\mathrm{pH}$-cyclying and after simulated toothbrushing.

Although, a smaller particle size of composite has been traditionally linked to smoother surfaces, Kaizar et al. (2014) systematically reviewed the literature comparing microhybrid and nanocomposites regarding different polishing procedures and surface challenges. Filtek Supreme XT was compared 35 times and presented varied results in relation to microhybrids. In $63 \%$ of the comparisons, Filtek Supreme was not different from the microhybrids, whereas it performed better than microhybrids in $11 \%$ and poorer in $26 \%$ of the comparisons. This was in support of Gönülol and Yilmaz (2012) who concluded that composites with smaller filler size did not necessarily show lower surface roughness.

According to Bollen et al. (1997), the clinically acceptable threshold value of surface roughness for composite resin restorations is $0.2 \mu \mathrm{m}$. Over this value, there would be an increase in plaque accumulation, risk of caries, and periodontal inflammation. On the other hand, Weitman and Eames (1975) reported that there were no differences in plaque accumulation throughout the roughness range of $0.7-1.4 \mu \mathrm{m}$. In the present study, at baseline, the average surface roughness values for both composite materials were less than $0.2 \mu \mathrm{m}$. Hence, using composite against the matrix strip produced the smoothest surface with a low risk for plaque accumulation.

At baseline, ANOVA revealed a significant difference between the different immersion solutions for both groups. However, pairwise test showed intergroup significance in Filtek Z250 only, but not for Filtek Supreme group. This unlikely finding at the baseline measurements can be explained statistically by random chance while grouping.

After 15 days and 30 days, there was a significant difference between the different immersion solutions in both groups. Specimens immersed in Coca-Cola had the roughest surface, followed by those immersed in orange juice, while 
specimens immersed in distilled water had the least surface roughness. The different values of surface roughness can be explained by the erosive effect of the immersion liquids due to the different values of their $\mathrm{pH}$ (Seow and Thong 2005).

The increase in surface roughness of both materials immersed in distilled water over time can be explained by the interaction of distilled water ( $\mathrm{pH}$ 6.9) with carbon dioxide in the atmosphere. Carbon dioxide dissolves into the distilled water, forming carbonic acid. Distilled water does not contain natural buffers; therefore the $\mathrm{pH}$ can drift as low as 5.0 (Calix et al. 2008). Münchow et al. (2014) explained that surface roughness of composite is a result of water sorption. Water softens polymer and hydrolyses the bonds within. This leads to leaching out of unbounded components.

In the present study, composite discs were in contact with the undiluted staining solutions. However, in the oral cavity, saliva dilutes and buffers all oral liquids. Further studies on this topic could mix the staining solutions with a medium that would represent saliva. All specimens were stored in the staining solution at $37^{\circ} \mathrm{C}$. Although this is appropriate for a controlled laboratory situation, it does not replicate oral conditions. Thermocycling could be incorporated to simulate the oral environment.

Although the contact surface evaluation is widely accepted, it may damage the specimens as the diamond stylus directly traces the surface. The more recent laser profilometry provides a 3D surface analysis at nanometer level without contacting the surface (Cazzaniga et al. 2015).

It is difficult to replicate all clinical conditions in a laboratory study with high precision; therefore, further clinical investigations are necessary to predict the longevity and acceptability of tooth coloured restorative materials.

\section{Conclusions}

From the present in vitro study, it can be concluded that:

Filtek Z250 and Filtek Supreme showed no significant difference in surface roughness and colour change at all measurement times.

There was a significant increase in surface roughness and colour change over time.

Specimens immersed in Coca-Cola demonstrated the greatest effect, followed by those immersed in orange juice, while specimens immersed in distilled water had the least effect.

Within the scope of this study, dentists can use Filtek Z250 and Filtek Supreme interchangeably in restorations. Moreover, parents and paediatric patients should be educated about the effect of soft drinks on the longevity of restorations, as well as, the correct preventive oral hygiene measures.
Funding This study was self funded by the corresponding author, Elwardani G.

\section{Compliance with ethical standards}

Conflict of interest All authors declare no conflict of interest.

Ethical approval This article does not contain any studies with human participants or animals performed by any of the authors.

Open Access This article is distributed under the terms of the Creative Commons Attribution 4.0 International License (http://creativeco mmons.org/licenses/by/4.0/), which permits unrestricted use, distribution, and reproduction in any medium, provided you give appropriate credit to the original author(s) and the source, provide a link to the Creative Commons license, and indicate if changes were made.

\section{References}

Al-Shalan TA. In vitro staining of nanocomposites exposed to cola beverage. Pak Oral Dent J. 2009;29(1):79-84.

Badra VV, Faraoni JJ, Ramos RP, Palma-Dibb RG. Influence of different beverages on the microhardness and surface roughness of resin composites. Oper Dent. 2005;30(2):213-9.

Bansal K, Acharya SR, Saraswathi V. Effect of alcoholic and nonalcoholic beverages on color stability and surface roughness of resin composites: an in vitro study. J Conserv Dent. 2012;15(3):283-8.

Bollen CM, Lambrechts P, Quirynen M. Comparison of surface roughness of oral hard materials to the threshold surface roughness for bacterial plaque retention: a review of the literature. Dent Mater. 1997;13(4):258-69.

Calix TF, Ferrentino G, Balaban MO. Measurement of high pressure carbon dioxide solubility in orange juice, apple juice, and model liquid foods. J Food Sci. 2008;73(9):e439-45.

Cazzaniga G, Ottobelli M, Ionescu A, Garcia-Godoy F, Brambilla E. Surface properties of resin-based composite materials and biofilm formation: a review of the current literature. Am J Dent. 2015;28(6):311-20.

Celik C, Yuzugullu B, Erkut S, Yamanela K. Effects of mouth rinses on color stability of resin composites. Eur J Dent. 2008;2(4):247-53.

Çelık EU, Aladağ A, Türkün L, Yilmaz G. Color changes of dental resin composites before and after polymerization and storage in water. J Esthet Restor Dent. 2011;23(3):179-88.

Commission Internationale de L'Eclairage. Colorimetry, official recommendations of the international Commission Illumination. Paris: Bureau Central de La CIE. Publication CIE No. 15 (E1.3.1), 1971.

Curtin JA, Lu H, Milledge JT, Hong L, Peterson J. In vitro staining of resin composites by liquids ingested by children. Pediatr Dent. 2008;30(4):317-22.

Curtis AR, Shortall AC, Marquis PM, Palin WM. Water uptake and strength characteristics of a nanofilled resin-based composite. J Dent. 2008;36(3):186-93.

de Moraes RR, Marimon JL, Schneider LF et al. Effects of 6 months of aging in water on hardness and surface roughness of two microhybrid dental composites. J Prosthodont. 2008;17(4):323-6.

Dietschi D, Campanile G, Holz J, Meyer JM. Comparison of the color stability of ten new-generation composites: an in vitro study. Dent Mater. 1994;10(6):353-62. 
Donly KJ, Dean JA. Restorative dentistry. In: Dean JA, editor. McDonald and Avery's dentistry for the child and adolescent, 10th ed. Oxford: Elsevier; 2016. pp. 185-205.

Erdemir U, Yildiz E, Eren MM, Ozel S. Surface hardness evaluation of different composite resin materials: influence of sports and energy drinks immersion after a short-term period. J Appl Oral Sci. 2013;21(2):124-31.

Ertaş E, Güler AU, Yücel AC, Köprülü H, Güler E. Color stability of resin composites after immersion in different drinks. Dent Mater J. 2006;25(2):371-6.

Ferracane JL. Hygroscopic and hydrolytic effects in dental polymer networks. Dent Mater. 2006;22(3):211-22.

Field A. Discovering statistics using IBM SPSS statistics, 4th ed. Thousand Oaks: Sage; 2013.

Frary CD, Johnson RK, Wang MQ. Children and adolescents' choices of foods and beverages high in added sugars are associated with intakes of key nutrients and food groups. J Adolesc Health. 2004;34(1):56-63.

Galal OM. The nutrition transition in Egypt: obesity, undernutrition and the food consumption context. Public Health Nutr. 2002;5(1):141-8.

Gerhardt K, Silva AS, Rego G et al. Bulk and surface properties related to composite filler size. Braz J Oral Sci. 2013;12:323-9.

Ghiorghe CA, Iovan G, Topoliceanu C, Sandu AV, Andrian S. Comparative study regarding the colorimetric changes of two composite resins after immersion in several beverages and one antibacterial mouthwash. Revista de Chemie. 2013;64(12):1436-40.

Gönülol N, Yılmaz F. The effects of finishing and polishing techniques on surface roughness and color stability of nanocomposites. J Dent. 2012;40:e64-70.

Hotwani K, Thosar N, Baliga S. Comparative in vitro assessment of color stability of hybrid esthetic restorative materials against various children's beverages. J Conserv Dent. 2014;17(1):70-4.

IBM Corp. SPSS statistics for windows, version 21.0. Armonk, NY, 2012.

Ionta FQ, Mendonça FL, de Oliveira GC et al. In vitro assessment of artificial saliva formulations on initial enamel erosion remineralization. J Dent. 2014;42(2):175-9.

Joiner A. Tooth color: review of the literature. J Dent. 2004;32:3-12.

Kaizer MR, de Oliveira-Ogliari A, Cenci MS, Opdam NJ, Moraes RR. Do nanofill or submicron composites show improved smoothness and gloss? A systematic review of in vitro studies. Dent Mater. 2014;30:e41-78.

Kim J, Paravina R, Chen JW. In vivo evaluation of color of primary teeth. Pediatr Dent. 2007;29(5):383-6.

Korkmaz Y, Ozel E, Attar N, Aksoy G. The influence of one-step polishing systems on the surface roughness and microhardness of nanocomposites. Oper Dent. 2008;33(1):44-50.

Lepri CP, Palma-Dibb RG. Surface roughness and color change of composite: Influence of beverages and brushing. Dent Mater J. 2012;31(4):689-96.

Mitra SB, Wu D, Holmes BN. An application of nanotechnology in advanced dental materials. J Am Dent Assoc. 2003;134(10):1382-90.

Mohan M, Shey Z, Vaidyanathan J et al. Color changes of restorative materials exposed in vitro to cola beverage. Pediatr Dent. 2008;30(4):309-16.
Münchow EA, Ferreira AC, Machado RM et al. Effect of acidic solutions on the surface degradation of a micro-hybrid composite resin. Braz Dent J. 2014;25(4):321-6.

Nagem Filho H, D’Azevedo MT, Nagem HD, Marsola FP. Surface roughness of composite resins after finishing and polishing. Braz Dent J. 2003;14(1):37-41.

Omata Y, Uno S, Nakaoki Y et al. Staining of hybrid composites with coffee, oolong tea, or red wine. Dent Mater J. 2006;25(1):125-31.

Oysaed H, Ruyter IE. Water sorption and filler characteristics of composites for use in posterior teeth. J Dent Res. 1986;65(11):1315-8.

Penteado RA, Tonholo J, Júnior JG et al. Evaluation of surface roughness of microhybrid and nanofilled composites after $\mathrm{pH}-$ cycling and simulated toothbrushing. J Contemp Dent Pract. 2010;11(6): $17-24$

Pierce CA, Block RA, Aguinis H. Cautionary note on reporting etasquared values from multifactor ANOVA designs. Educ Psychol Meas. 2004;64(6):916-24.

Poggio C, Ceci M, Beltrami R et al. Color stability of esthetic restorative materials: a spectrophotometric analysis. Acta Biomater Odontol Scand. 2016;2(1):95-101.

Powers JM, Wataha JC, Chen Y. Direct esthetic restorative materials. In: Powers JM, Wataha JC, editors. Dental materials: foundations and applications. 11th ed. Oxford: Elsevier; 2017. pp. 42-58.

Samorodnitzky-Naveh GR, Geiger SB, Levin L. Patients' satisfaction with dental esthetics. J Am Dent Assoc. 2007;138(6):805-8.

Sarac D, Sarac YS, Kulunk S, Ural C, Kulunk T. The effect of polishing techniques on the surface roughness and color change of composite resins. J Prosthet Dent. 2006;96(1):33-40.

Sarafianou A, Iosifidou S, Papadopoulos T, Eliades G. Color stability and degree of cure of direct composite restoratives after accelerated aging. Oper Dent. 2007;32(4):406-11.

Seow WK, Thong KM. Erosive effects of common beverages on extracted premolar teeth. Aust Dent J. 2005;50(3):173-8.

Sideridou I, Tserki V, Papanastasiou G. Effect of chemical structure on degree of conversion in light-cured dimethacrylate-based dental resins. Biomaterials. 2002;23(8):1819-29.

Söderholm KJ, Zigan M, Ragan M, Fischlschweiger W, Bergman M. Hydrolytic degradation of dental composites. J Dent Res. 1984;63(10):1248-54.

Topcu FT, Sahinkesen G, Yamanel K et al. Influence of different drinks on the colour stability of dental resin composites. Eur J Dent. 2009;3(1):50-6.

Turssi CP, Hara AT, Serra MC, Rodrigues AL. Effect of storage media upon the surface micromorphology of resin-based restorative materials. J Oral Rehabil. 2002;29(9):864-71.

Uchimura JY, Sato F, Bianchi G et al. Color stability over time of three resin-based restorative materials stored dry and in artificial saliva. J Esthet Restor Dent. 2014;26(4):279-87.

Venz S, Dickens B. NIR-spectroscopic investigation of water sorption characteristics of dental resins and composites. J Biomed Mater Res. 1991;25(10):1231-48.

Weitman RT, Eames WB. Plaque accumulation on composite surfaces after various finising procedures. J Am Dent Assoc. 1975;91(1):101-6. 